\title{
ENDOFUNCTORS OF QUANDLES AND RACKS
}

\author{
PETER ULRICKSON
}

\begin{abstract}
We show that the only endofunctors of the category of quandles commuting with the forgetful functor to sets are the power operations. We also give a similar statement for racks.
\end{abstract}

\section{INTRODUCTION}

Given a quandle $(X, \triangleright)$ the assignments $(X, \triangleright) \mapsto\left(X,(x, y) \mapsto x \triangleright^{n} y\right)$ for integers $n$ yield endofunctors of the category of quandles. Here $x \triangleright^{n} y$ denotes the element obtained through the $n$-fold application of $x \triangleright-$, i.e. the element $x \triangleright(x \triangleright(x \ldots(x \triangleright y) \ldots))$. In [6] it is noted that these functors, referred to as power operations, commute with the forgetful functor to sets and thus correspond to endofunctors of the algebraic theory of quandles. A question left open there is what other set-preserving endofunctors of the category of quandles there might be. In fact, these power operations are the only such endofunctors.

Remark 1.1. Lawvere [3] describes the correspondence between functors of categories of algebras for algebraic theories which preserve underlying sets and functors between algebraic theories. See also [1], which refers to such underlying set preserving functors as Lawvere functors. Thus we may speak of endofunctors of the algebraic theory of quandles while in fact working with endofunctors of the category of quandles (or racks) preserving underlying sets.

Remark 1.2. In [5] the power operations are treated in a more general setting. There, families of mutually distributive operations are considered. Converting from right to left distributivity, their Lemma 1 notes the requisite left self-distributivity of a composition of a left self-distributive operation with itself.

Recall that any group $G$ has an associated quandle known as the conjugation quandle of $G$. The underlying set of this quandle is the underlying set of the group, endowed with the operation $x \triangleright y:=x y x^{-1}$.

Remark 1.3. There are two conventions for quandles and racks. The one we follow here is that of [6], where $\triangleright$ is left self-distributive and 
each $x \triangleright-$ is bijective. Others, including [5], consider operations * which are right self-distributive and such that each $-* x$ is bijective.

\section{Free Quandles and Conjugation Quandles of Free Groups}

There is a free functor from sets to quandles which is a left adjoint to the functor which forgets the binary operation. One construction of the free quandle on a given generating set is by combining elements of the set using the symbols $\triangleright$ and $\triangleright^{-1}$, considering such words modulo equivalence by the requisite relations for quandles. The symbol $\triangleright^{-1}$ is the binary operation satisfying $x \triangleright\left(x \triangleright^{-1} y\right)=y$ for all $x$ and $y$ in the generating set. Note that self-distributivity of $\triangleright^{-1}$ is the same as that for $\triangleright$. Moreover, the two are mutually-distributive in the terminology of [5], as in the following equation.

$$
x \triangleright\left(y \triangleright^{-1} z\right)=(x \triangleright y) \triangleright^{-1}(x \triangleright z)
$$

Elements in a free quandle can be put in a standard form. Given an element $q$ in the free quandle, and an element $x$ of the generating set, we refer to elements $x \triangleright q$ and $x \triangleright^{-1} q$ as left multiplications by $x$.

Lemma 2.1. Every element in a free quandle can be written as a composition of left multiplications by generating elements.

Proof. First, observe the following equality.

$$
(x \triangleright y) \triangleright-=x \triangleright\left(y \triangleright\left(x \triangleright^{-1}-\right)\right)
$$

Repeating such a rewriting as necessary, it follows that multiplying on the left by any composition of left multiplications is equivalent to applying a composition of left multiplications.

The case considered above shows that all elements of the free quandle involving only two operations can be written as a composition of left multiplications. By induction on the number of operations one sees that every element can be expressed as a composition of left multiplications by generating elements.

We now relate endofunctors of the algebraic theory of quandles with elements of the free quandle on a two-element generating set. This was noted in Remark 5.5 of [6].

Lemma 2.2. Endofunctors of the algebraic theory of quandles are determined by their restriction to the free quandle on a set with two elements. 
Proof. Once the new product $x * y$ is determined for the free quandle on two elements $x$ and $y$, the products on all quandles are determined, since for any pair of elements $a$ and $b$ in any quandle there is a unique quandle morphism extending $x \mapsto a, y \mapsto b$ and the functor must leave underlying set maps unchanged.

Given an element $w$ in the free quandle on $\{x, y\}$ we now produce two elements in the free group on $\left\{x, y_{1}, y_{2}\right\}$. Let $g_{i}$ be the morphism from the free quandle on $\{x, y\}$ to the conjugation quandle of the free group on $\left\{x, y_{1}, y_{2}\right\}$ determined by the set map sending $x$ to $x$ and $y$ to $y_{i}$ for $i=1,2$. Then two group elements, denoted $w_{1}$ and $w_{2}$, are determined by $w_{i}:=g_{i}(w)$.

Lemma 2.3. If $w_{1} w_{2}^{-1}$ cyclically reduces to $y_{1} y_{2}^{-1}$, then $w_{1}$ is $x^{k} y x^{-k}$ for an integer $k$.

Proof. The element $w_{1}$ is represented by a word with the form

$$
x^{e_{1}} y_{1}^{f_{1}} x^{e_{2}} \ldots x^{e_{k}} y_{1} x^{-e_{k}} \ldots y_{1}^{-f_{1}} x^{-e_{1}}
$$

and thus $w_{1} w_{2}^{-1}$ takes the form

$$
x^{e_{1}} y_{1}^{f_{1}} x^{e_{2}} \ldots x^{e_{k}} y_{1} x^{-e_{k}} \ldots y_{1}^{-f_{1}} x^{-e_{1}} x^{e_{1}} y_{2}^{f_{1}} x^{e_{2}} \ldots x^{e_{k}} y_{2}^{-1} x^{-e_{k}} \ldots y_{2}^{-f_{1}} x^{-e_{1}}
$$

which cyclically reduces to

$$
y_{1}^{f_{1}} x^{e_{2}} \ldots x^{e_{k}} y_{1} x^{-e_{k}} \ldots y_{1}^{-f_{1}} y_{2}^{f_{1}} x^{e_{2}} \ldots x^{e_{k}} y_{2}^{-1} x^{-e_{k}} \ldots y_{2}^{-f_{1}}
$$

If $f_{1}$ is non-zero this word is cyclically reduced, and hence cannot yield $y_{1} y_{2}^{-1}$. Thus $w_{1}$ must be of the form $x^{k} y_{1} x^{-k}$.

We now determine the elements in the free quandle that lead to free group elements of the form considered in the previous construction.

Lemma 2.4. Let $w$ be an element of the free quandle on $\{x, y\}$. If the image of $w$ under the morphism to the conjugation quandle of the free group on $\{x, y\}$ induced by the identity map on generating sets has the form $x^{k} y x^{-k}$ for an integer $k$, then $w=x \triangleright^{k} y$.

Proof. The element $w$ may be written as a composition of left multiplications, as in Lemma 2.1, so that we have

$$
w=z_{1} \triangleright^{e_{1}}\left(z_{2} \triangleright^{e_{2}}\left(z_{3} \triangleright^{e_{3}}\left(\ldots \triangleright\left(z_{m} \triangleright^{e_{m}} y\right)\right)\right) \ldots\right)
$$

The exponents $e_{i}$ are either 1 (yielding the ordinary operation) or -1 (yielding the inverse operation). The terms $z_{i}$ are either $x$ or $y$. The element in the free group on $x$ and $y$ produced from such a quandle element is represented by $\left(\Pi z_{i}^{e_{i}}\right) y\left(\Pi z_{i}^{-e_{i}}\right)$, and such a word is equivalent to $x^{k} y x^{-k}$ only if any extraneous initial occurences of $y$ are eliminated by adjacent occurrences of $y$ with exponent of opposite sign. 


\section{Classification of Endofunctors}

A one-relator group is a group with a presentation $\langle S \mid\{r\}\rangle$ where the set of relations consists of a single relation $r$. Magnus [4] showed the following, referred to as the Freiheitssatz, for one-relator groups.

Theorem 3.1 (Freiheitssatz). Suppose that $\left\langle\left\{a_{1}, \ldots, a_{n}, x\right\} \mid\{r\}\right\rangle$ is a presentation of a group $G$ with a single relation $r$. If $x$ cannot be eliminated from $r$ through cyclic reduction, then $\left\{a_{1}, \ldots, a_{n}\right\}$ generates a free subgroup in $G$.

By constructing suitable one-relator groups we can use this theorem to exclude certain operations as possible endofunctors of the category of quandles.

Theorem 3.2. The only endofunctors of the algebraic theory of quandles are the power operations.

Proof. As was already noted in Lemma 2.2, such an endofunctor is determined by its restriction to the free quandle on $\{x, y\}$. Let $w$ be an element in this free quandle. Produce elements $w_{1}$ and $w_{2}$ in the free group on $\left\{x, y_{1}, y_{2}\right\}$ as above, and consider the one-relator group $G$ with presentation $\left\langle\left\{x, y_{1}, y_{2}\right\} \mid\left\{w_{1} w_{2}^{-1}\right\}\right\rangle$.

Suppose that a cyclically reduced expression for $w_{1} w_{2}^{-1}$ contains all three generators. By the Freiheitssatz the set $\left\{y_{1}, y_{2}\right\}$ generates a free subgroup, so that $y_{1}$ and $y_{2}$ are distinct. Use $w$ to produce a new binary operation $*$ on the (underlying set of the) conjugation quandle of $G$. This operation fails to define a quandle, since $x * y_{1}=x * y_{2}$ even though $y_{1} \neq y_{2}$. Thus $w$ does not yield an endofunctor of the algebraic theory of quandles.

If $w_{1} w_{2}^{-1}$ contains only $y_{1}$ and $y_{2}$ then it is equivalent to $y_{1} y_{2}^{-1}$, and Lemma 2.4 states that $w$ is of the form $x \triangleright^{k} y$, which gives a power operation.

The same reasoning which led to the previous theorem also allows for the classification of endofunctors of the algebraic theory of racks. Recall that a rack is a set with a binary operation $\triangleright$ which satisfies the same left self-distributivity condition as for quandles and for which $x \triangleright-$ is bijective. What is not required is that $x \triangleright x=x$. There is a forgetful functor from quandles to racks whereby we view the conjugation quandle of a group as a rack.

Just as in the case of quandles, the distributivity and bijectivity mean that one can write general elements of the free rack on two elements as a composition of left multiplications using $\triangleright$ and $\triangleright^{-1}$. A word $w$ in the free rack determining an endofunctor cannot contain terms of the form 
$y \triangleright x$ by the same Freiheitssatz argument. On the other hand, terms of the form $y \triangleright y$ cannot be reduced in a free rack, so that we have the following classification.

Theorem 3.3. The only endofunctors of the algebraic theory of racks are of the form $x \triangleright^{k} F^{j}(y)$, where $F: y \mapsto y \triangleright y$ is the canonical automorphism of a rack and $k$ and $j$ are integers.

Remark 3.4. As was observed in the remark of [6] raising the question addressed here, Kan [2] showed that the algebraic theory of groups admits only the identity endofunctor.

Acknowledgements: I thank M. Szymik for his comments on a draft of this note.

\section{REFERENCES}

[1] P. Freyd. Algebra valued functors in general and tensor products in particular. Colloq. Math., 14:89-106, 1966.

[2] Daniel M. Kan. On monoids and their dual. Bol. Soc. Mat. Mexicana (2), 3:52$61,1958$.

[3] F. William Lawvere. Functorial semantics of algebraic theories. Proc. Nat. Acad. Sci. U.S.A., 50:869-872, 1963.

[4] Wilhelm Magnus. Über diskontinuierliche Gruppen mit einer definierenden Relation. (Der Freiheitssatz). J. Reine Angew. Math., 163:141-165, 1930.

[5] Józef H. Przytycki and Adam S. Sikora. Distributive products and their homology. Comm. Algebra, 42(3):1258-1269, 2014.

[6] Markus Szymik. Permutations, power operations, and the center of the category of racks. Comm. Algebra, 0(0):1-11, 2017, http://dx.doi.org/10.1080/00927872.2017.1316857.

Department of Mathematics, The Catholic University of America, WASHINGTON, DC

E-mail address: ulrickson@cua.edu 